\title{
Integrando Facebook en el Modelo de Educación Deportiva: Una experiencia práctica Integrating Facebook in Sport Education: A practical experience
}

Javier Bernabé Martín, Francisco Javier Fernández-Río IES Real Instituto de Jovellanos de Gijón (España), Universidad de 0 viedo (España)

\begin{abstract}
Resumen. El aumento del uso educativo de las redes sociales se debe aque ofrecen recursos que facilitan tanto el seguimiento de la actividad académica como los aspectos sociales inherentes al proceso educativo; sin embargo, estas plataformas no son empleadas habitualmente como un recurso para la educación. Facebook ofrece diversos canales de comunicación y ha mostrado su potencialidad para vehicular las actividades colaborativas entre estudiantes. En esta experiencia se empleó Facebook como entorno para desarrollar las características del modelo de educación deportiva (temporada, afiliación, competición formal, registro sistemático, ambiente festivo y celebración final) en el área de Educación Física, empleando los recursos propios de lared social. Cadaunade lascaracterísticas del modelo pedagógico se desarrolló através delas herramientas de la red social en un grupo creado como recurso complementario a la acción educativa de aula. Los progenitores del alumnado participante se inscribieron y pudieron participar a distancia en la actividad, que se extendió fuera del centro educativo graciasal empleo de Facebook. Losresultadosmuestran un mayor seguimiento, implicación parental y laconstrucción de una comunidad de práctica. El modelo educativo utilizado pudo desarrollarse de forma más completa, logrando que el alumnado se mantuviera pendiente de la actividad fuera del centro educativo.
\end{abstract}

Palabras clave: redes sociales, comunidades de práctica, secundaria, educación física.

Abstract. The increased educational use of social networks is due to the fact that they offer resources that facilitate both the monitoring of academic activity and the social aspects inherent in the educational process; however, these platforms are not usually used as a resource for education. Facebook offers various channels of communication and has shown its potential for channeling collaborative activities between students. In this experience, Facebook was used as an environment to develop the characteristics of the sportseducation model (season, affiliation, formal competition, systematic registration, festive atmosphere and final celebration) in the area of Physical Education, using the resources of the social network. Each of the characteristics of the educational model was developed through the tools of the social network in a group created as a complementary resource to the educational action in the classroom. The parents of the participating students registered and were able to participate in the activity from a distance, which was extended outside the school thanks to the use of Facebook. The results showed increased monitoring, parental involvement, and the building of a community of practice. The educational model used was fully developed, helping the students «keep an eye» on the activity outside the school.

Keywords: social networks, learning communities, secondary school, physical education.

\section{Introducción}

Las redes sociales son cada vez más empleadas como recurso educativo, y su uso supone un cambio en la formación de laspersonas(Balogh, M unk, \&Turcani, 2011), ya que fomentan el intercambio práctico de informa ción entrelos participantes, organizan el trabajo en grupo y mejoran la comunicación con el profesorado; estos factores favorecen la adquisición de competencias relacionales y de un capital social en la red social, (Erjavec, 2013) que se expresa en la construcción de un grupo con intereses comunes a partir del objetivo de

Fecha recepción: 30-08-20. Fecha de aceptación: 06-02-21

Javier Bernabé Martín

jvbernabe@ gmail.com realizar la tarea propuesta. Además las redes sociales benefician la autonomía del al umnado ya que fomentan la colaboración extraescolar, la ayuda extra en los deberes, la discusión académica y la autoorganización (Fewkes \& McCabe, 2012). Por último, se posibilita la creación de una red contextualizada a medida de cada usuario (Madge, Meek, Wellens, \& Hooley, 2009). Sin embargo, las aulas aún permanecen, básicamente, aisladas sobre todo si se habla de la introducción y uso de las redes sociales y no tanto de la tecnología, que es usada en el entorno educativo como recurso material, pero en general con poco sentido pedagógico (Casey, Goodyear, \& Armour, 2016)

Facebook es una empresa que ofrece servicios de redes sociales en línea. Fue creada en 2004. En el momento de su creación Facebook se definía como: «Un 
directorio en línea que conecta a las personas a través de las redes sociales en colegios y universidades»(Facebook, s.f. ). La empresa abandonó la intención de ser un recurso educativo propiamente dicho después de lanzar facebook Groups for School, una utilidad que encontró problemas al dictar el Departamento de Educación de Q ueensland (Q ueensland, 2018) que las redes sociales debían establecerse como sitios oficiales para toda la escuela y no a través de grupos separados; lo cual cortaba de raíz la posibilidad de desarrollar una herramienta como Groups for School a un nivel de uso de toda una comunidad educativa.

U na pregunta que hay que responder para satisfacer las demandas del ámbito educativo es si Facebook puede ser una herramienta de aprendizaje. 0 tra cuestión que responder es cómo puede ser utilizada. Un modelo de pedagogía de Facebook tendría que considerar, además de las características del potencial alumnado y de los usos que se hacen de Facebook, las características de la propia herramienta y queda justificado desde el momento que se entiende que las redes sociales cambian el modo en el que se representa la comunicación (Kontopodis, Varvantakis, \& Wulf, 2017) ya que la escritura no es el principal ni el único medio de producir significado (Bezemer \& K ress, 2017). Estosautores proponen una «pedagogía de la elaboración de textos multimodales» (p. 6), que incluya en los curricula escolares la forma real de expresión en Facebook como forma de texto.

La investigación que examina este entorno virtual como herramienta de enseñanza 0 aprendizaje lo hace desde diversos enfoques, que se pueden resumir en cinco (N iu, 2017): Facebook como entorno de aprendizaje (Akbari, Pilot, \& Simons, 2015); como plataforma de discusión (Kent, 2016; Wu \& Chen, 2015); Facebook como extensión de un curso o materia (Dzvapatsva, Mitrovic, \& Dietrich, 2014; English \& Duncan-Howell, 2008); Facebook como canal de información (Mena et al. , 2012; O mar, Embi, \& M dYunus, 2012) y Facebook como Learning M anagement System (LMS) (M eisharTal, Kurtz, \& Pieterse, 2012).

Facebook ofrece enormes posibilidades de comunicación a través de la capacidad de añadir notas o comentar ios a texto, fotos, videoso hipervínculos o hacer chats en vivo (Alhenshiri, Badesh, \& Stephanidis, 2015), usando herramientas como la mensajería instantánea, tablones de anuncios y email que permiten la actividad multitarea (Junco, 2013), además de proporcionar otras actividades como ver fotos o chatear con otros miembros sin salir del sitio ofreciendo múltiples posibilidades de ex- plorar y actuar (J unco \& Cotten, 2012); sin embargo, su estructura puede resultar confusa debido a la cantidad de herramientas que ofrece de manera simultánea y a la posibilidad de usar canales diferentes para comunicarse (Wang, 2018), haciendo de Facebook un lugar particularmente distractor (Wood et al., 2012).

\section{El Modelo de Educación deportiva}

Partiendo de la evidencia que ha mostrado que Facebook puede promover la participación en activida des colaborativas (Selwyn \& Stirling, 2016) se planteó utilizar la red social en combinación con el Modelo de Educación Deportiva (MED) (Siedentop, 1994). Este modelo se basa en el concepto de que grupos de trabajo pequeños y compuestos por estudiantes con diferentes habilidades, a los que se llama genéricamente «equipos», trabajen juntos de forma que todos sus componentes se beneficien y experimenten el éxito (Siedentop, Hastie, \& Van Der Mars, 2019).

Las metas que se propone el MED se articulan en torno a educar a los estudiantes ayudándoles a desarroIlarse como deportistas competentes, cultos y entusiastas; entendiendo que el al umnado ha de ser competente para participar en las actividades físicas desarrollando las habilidades requeridas suficientemente, ha de ser culto para apreciar los valores, reglas y tradiciones de las actividades y ha de ser entusiasta en el sentido de que han de tener la motivación para querer participar en las actividades físicas o deportivas porque han llegado a valorar las experiencias y el disfrute que se deriva de la práctica (Siedentop et al., 2019).

EI MED organiza su propuesta señalando unas características clave que le diferencian de los enfoques tradicionales de la docencia de la EF, y que son los siguientes (Siedentop et al., 2019): (1) Temporada. La unidad didácticarepresentaunatemporada deportivareal y cuenta con mayor duración que las unidades didácticas tradicionales. (2) Afiliación. Los estudiantes forman parte de equipos reducidos que permanecen fijos durante la temporada desde la primera sesión. (3) Competición formal. Desde el principio de la unidad se organiza una competición intercal ada con las actividades de aprendizaje (ejercicios y juegos modificados). (4) Registro sistemático. El registro del rendimiento de los estudiantes permite motivarles, darles feedback, evaluar o construir estándares. (5) Ambiente festivo. La temporada debe ser festiva; es decir, se intenta celebrar el éxito obtenido por los estudiantes. Por lo tanto, se diseñarán actividades para que el alumnado alcance un nivel de 
motivación a través del éxito en la actividad (Cecchini, González, M éndez-Giménez, \& Fernández-Río, 2011). (6) Evento culminante. EI MED culmina con un evento que debe cerrar la actividad y reconocer todo el trabajo desarrollado a lo largo de la temporada. (7) Por último, los roles son un elemento característico del MED que concreta el desarrollo de la actividad. A través de ellos, los estudiantes aprenden a planificar y administrar su experiencia deportiva desempeñando diversas funciones que dependerán de la actividad que se proponga, conforme a la naturaleza del contenido a tratar.

EI MED es uno de los modelos pedagógicos para la EF más extendidos y que más producción investigadora ha suscitado. Las sucesivas revisiones que se han realiza do (Guijarro, Rocamora, Evangelio, \& GonzálezVíllora, 2020; Hastie, de 0 jeda, \& Calderón, 2011; Wallhead \& 0 'Sullivan, 2005) muestran tanto la expansión del MED como su adaptación a diversos contextos ( $M$ enendezSanturio \& Fernández-Río, 2016) y contenidos (Puente-Maxera, Méndez-Giménez, \& Martínez de 0 jeda, 2020).

\section{Facebook y Modelo de Educación Deporti- va}

La literatura recoge experiencias de uso de redes sociales en combinación con el MED. La red Edmodo fue empleada por Gutierrez, Segovia, Gárcia y Fernández (2019) y la red social Twitter fue usada por André (2018). La red social Facebook ha sido empleada en el ámbito del deporte de club (Luguetti, Goodyear, $\&$ André, 2019) y como recurso complementario en la EF escolar (Fernandez-Rio \& Bernabe-Martín, 2019). Facebook ha sido usado para el aprendizaje de la peda gogía deportiva (Margarit, Tonita, \& Angelescu, 2018) o para la mejora de habilidades técnicas (Palade, Gheorghe, Ciolca, \& Badea, 2018). 0 tras iniciativas de uso de Facebook se han centrado en el desarrollo de programas para promoción de la salud (Maher et al., 2014; N apolitano, Hayes, Bennett, Ives, \& Foster, 2013).

Teniendo en cuentalas peculiaridades de Facebook y del MED, el objetivo principal que se trazó para la experiencia que se relata fue el de desarrollar las características del MED en la red social Facebook, en una unidad didáctica de EF escolar, usando la red social como recurso educativo complementario a la acción de aula. De este objetivo principal se derivan otros dos específicos: (1) aumentar la participación del alumnado en la organización y realización de los aspectos de la actividad y (2) mejorar el conocimiento de la actividad más allá del tiempo de clase en el centro educativo, en el sentido de «hacer más con menos tiempo» descrito por André (2018, p. 122), teniendo en cuenta que la dificultad practica de tiempo en la EF, hace difícil conseguir losobjetivos delamateria(Hardman, 2008; M ohammed, 2016; Sánchez-Bañuelos, 1997) y es necesario optimizar los recursos temporales disponibles. (2) Desarrollar en profundidad el objetivo principal del MED (Siedentop et al., 2019); es decir, la competencia en la actividad propuesta, la cultura que conlleva conocer lo que debe conseguir y valorarlo y el entusiasmo para practicar para hacerlo lo mejor posible.

\section{Planteamiento didáctico de la experiencia: Propuesta de integración Facebook-M odelo de Educación Deportiva}

En un Instituto de Educación Secundariay en el área de EF se desarrolló una unidad didáctica de 16 sesiones cuyo contenido era Acrosport dirigida a dos grupos de 30 de ESO de 23 y 25 estudiantes respectivamente. LoS estudiantes habían tomado contacto con el MED anteriormente desde el comienzo del mismo curso con otros contenidos, de forma que estaban familiarizados con aspectos como el trabajo en equipo, la asunción de roles, la puntuación y la competición. La experiencia del docente con el MED se remontaba a los cinco cursos previos y este mismo contenido lo había desarrollado en los tres cursos previos empleando este modelo. La actividad se llevó a cabo en el segundo trimestre del curso, a lo largo de 65 días en total. En la unidad didáctica se desarrolló el MED (Siedentop et al. , 2019), abordando cuatro competiciones intermedias organizadas en el formato «Round Robin», según el cual cada equipo se enfrenta al resto a lo largo de la competición; al final de la actividad se llevó a cabo una celebración final. Los estudiantes trabajaron en equipos estables a lo largo de toda la unidad y desarrollaron roles específicos que se concretaron en: anotador, responsable de material y seguridad, periodista y arbitraje.

Paralelamente al desarrollo de la actividad en el aula, se dispuso un grupo de Facebook en el cual se mostraba el desarrollo de esta actividad. La red Facebook se usó porque es la red más usada a nivel mundial (Statista, 2018) y que el acceso a esta red resulta más fácil y directo que a redes temáticas educativas, en las que el acceso tiene restricciones. El grupo tuvo carácter privado y fue creado por el docente que también se encargó de su administración y se dirigió únicamente a los progenitores del alumnado participante, que debían 
adscribirse al grupo después de cumplimentar un formulario de preguntas que acreditaran su identidad; una vez inscritos, podían participar en las actividades de dicho grupo. De los 97 padres y madres que podrían participar en el grupo de Facebook, se inscribieron un total de $41(42,26 \%)$, de los cuales, 28 fueron madres y 13 fueron padres. La propuesta presentada se apoyó en la idea replicar el MED en Facebook, incluyendo y desarrollando todas las características definitorias del modelo, usando todos los recursos que ofrece Facebook (Fewkes \& McCabe, 2012) a fin de proporcionar un nuevo canal de comunicación y de participación creando comunidades de aprendizaje que persigan objetivos comunes y los desarrollen (Luguetti et al., 2019).

En cuanto al procedimiento ético empleado en la experiencia, se observaron todos los relativos a la preserva ción de datos de tipo personal de todos los participantes: En primer lugar, se obtuvo el permiso del comité de ética de la universidad de los investigadores para llevar a cabo el proyecto de investigación que dio como fruto la experiencia que se narra a continuación. En segundo lugar, se contactó con el centro educativo objetivo de la investigación para explicar el proyecto, obteniendo el permiso de sus responsables. En tercer lugar, se contactó con el grupo de progenitores implica dos para solicitar su participación en el proyecto, obteniendo un consentimiento informado.

Por último, se informó al alumnado en la primera sesión de la creación de dicho grupo, al cual podían adherirse sus padres, sus madres o los dos progenitores y se repartió una carta explicativa para que la llevaran a casa. Se les comunicó que a lo largo de la unidad didáctica irían colgándose en este grupo de Facebook videos y fotos de las actividades que se llevasen a cabo. En todo momento se respetó lo recogido en la normativa vigente en relación a la protección de datos y su aplica ción en centros docentes (AEPD, 2018), para ello se elaboró previamente un informe que evaluó la idoneidad de la herramienta Facebook como recurso educativo para esta actividad; posteriormente ,y siguiendo las instrucciones del Servicio de Inspección Educativa de la Consejería de Educación y Cultura (2018) el uso de Facebook para esta actividad fue aprobado por la Comisión de Coordinación Pedagógica, órgano competente del centro educativo a este efecto.

Antes de habilitar el grupo de Facebook y de co- menzar la actividad en el aula, se requirió de una organización previa, en la que se pusieron a prueba los recursos de Facebook que, en principio, podrían usarse a Io largo de la experiencia, (encuestas, votaciones, enla ces, videos) a fin de comprobar su utilidad real; esta fase también sirvió de aprendizaje al docente para gestionar la administración del grupo. Se replicaron las características definitorias del MED, adaptándolas a su presencia en Facebook de la siguiente manera (Figura 1).

Temporada. Es la unidad temporal a lo largo de la cual se organiza la actividad. Se planificó con detalle para que la actividad de Facebook ayudará a difundir los objetivos y tareas desarrolladas, usando el muro de Facebook como un tablón de anuncios (Stutzman, 2006) y con el propósito de que Facebook fuera una plataforma de seguimiento de la actividad a lo largo del tiempo. Para conseguir estos dos fines - comunicación y seguimiento- se llevaron a cabo tres acciones: (a) Publicar la «agenda de la actividad»: La primera información que se mostraba al acceder al grupo de Facebook era el anuncio de la actividad que estaba en curso, señalando el momento de desarrollo en el que se estaba. El primer día se publicó el desarrollo previsto de la actividad. Se incluyó el número de sesiones, los días que estaban previstos para las competiciones y la fecha de la celebra ción final. En cada sesión se anticipaba el contenido previsto. A partir de la segunda sesión, cada día se anunciaba en el muro de Facebook lo que se estaba llevando a cabo en ese momento, a través de la herramienta de «anuncios» de Facebook, que permite seleccionar la primera información visible para el visitante al grupo. (b) Resúmenes semanales: Estos consistieron en videoclips de corta duración donde el docente informó de la actividad a través de un feedback previo o posterior a las clases de EF. Tuvieron una secuencia semanal; en el primer resumen se anticipaba lo que estaba previsto ha- 
cer, y en el segundo se detallaban los resultados y la actividad de la semana. A través de estos resúmenes se mantuvo el interés centrado en la actividad, fomentando su seguimiento diario a lo largo de todo el tiempo de duración de la actividad. (c) Por último, fue importante mantener una rutina temporal de publicaciones en toda la actividad que se generó en Facebook. Estas rutinas, fa cilitaron la orientación de los participantes, haciendo más fácil de localizar las publicaciones y permitieron anticipar el momento de publicación de los diferentes tipos de contenidos (puntuaciones, encuestas, fotos... ); ya que una de las debilidades de esta red social es lo confuso que puede resultar, sobre todo para quién se inicia (Wang, 2018). Por ejemplo, las clasificaciones se publicaban los fines de semana y las fotos y videos de las clases, después de la última clase de la semana.

2. Afiliación. La afiliación define el trabajo en pequeños grupos llamados equipos. El hecho de mantener a los estudiantes comprometidos con las tareas de las que son responsables promueve una práctica relevante a través de actividades de diferente carácter,(Romar, Sarén, \& Hastie, 2016) , por eso es importante que Facebook muestre las acciones. Las acciones previstas para adaptar el aspecto de afiliación a Facebook fueron las siguientes: (a) Publicación de la simbología de los equipos: A través de Facebook se contribuyó a la creación del sentimiento de equipo desde los primeros momentos, publicando de forma sistemática la afiliación de cada participante, el nombre de cada equipo en cada ocasión en la que aparecía un intento, ensayo o demostración de cualquier tarea desarrollada a lo largo de la actividad. También se promovió la afiliación haciendo que los participantes llevaran la equipación acordada (color de ca miseta, calcetines, muñequeras, etc.... ) y otros símbolos propios como banderolas, estandartes, mascotas, cuadernos etc. Facebook, en este sentido, fomentó que los equipos acentuaran los lazos que les identificaban (M enendez \& Fernandez-Rio, 2017) y ayudó a que compartieran lealtad a los símbolos significativos, mostrando como participaban juntosy acentuando el sentimiento de pertenecer a una comunidad única: el equipo. Esto favoreció la creación de lazos que persistieron a lo largo del tiempo; tal y como ocurre en las comunidades de aprendizaje (Wenger, 2011). (b) M ostrar la actividad de la clase de EF: Además de publicar los símbolos que formalmente caracterizaran a cada equipo, la afiliación en Facebook se mantuvo a lo largo de toda la temporada, utilizando los recursos multicanal de Facebook, entre loscuales ocupaun lugar destacado la posibilidad de transferir lo que ocurre en la clase a la red social a través de videoclips. Toda la actividad de clase se publicó en el muro de Facebook. La posibilidad de mostrar los clips se empleó de las siguientes formas: (1) Publicando las habilidades motrices del alumnado y permitiendo abordar el aprendizaje desde la observación, ya que los estudiantes prestaban atención a las habilidades motrices realizadas para reproducirlas y mejorar su autoeficacia (Bandura, 1997). (2) M ostrando losaspectosqueno eran estrictamente motrices desarrollados por los estudiantes, tales como el desempeño de roles como los descritos en el MED, cuyas acciones consistieron en anotar resultados, colocar y devolver material a su sitio, ser jueces sometidos a criterios que tuvieron que aplicar 0 , dependiendo de los roles que se implementaron, pudieron incluso entrevistar, explicar públicamente diversos aspectos de la actividad etc. Todos estos y otros momentos fueron publicados en Facebook; también se mostra ron clips de los equipos tomando decisiones conjuntas sobre soluciones tácticas grupales. Es decir, las publica ciones describieron el ambiente de trabajo en el aula, incluyendo los intentos del alumnado para conseguir dichas habilidades. La actividad sistemática de mostrar lo que aconteció en clase, fomentó el seguimiento de la actividad y el sentimiento de afiliación a los equipos; esto estuvo relacionado con los beneficios del uso del video en losprocesos de feedback (Kretschmann, 2017); como modelo ayuda a muchos estudiantes a mejorar sus habilidades (Zhang \& Li, 2018) y como recurso educativo enriquece el desarrollo de las experiencias de movimiento (Koekoek, van der M ars, van der Kamp, Walinga, $\&$ van Hilvoorde, 2018). Por esto, su uso a través de la red social Facebook refuerza la afiliación, las posibilida des de mejora del alumnado y la motivación hacia la práctica de las habilidades propuestas en la clase (Bernabe-Martín, 2018a). (3) Concursos relacionados con temas de salud y actividad física: A lo largo de toda la actividad se fueron publicando periódicamente concursos de diferente temática: adivinanzas, encontrar un lugar 0 señal de tráfico, hacerse una foto en determinado lugar 0 haciendo al guna actividad física concreta. La participación en estos concursos se llevó a cabo por parte de miembros de la misma familia, fomentando la afiliación al equipo (el mismo de clase) o por miembros de los mismos equipos fuera de clase, favoreciendo, de nuevo, el sentimiento de pertenencia al equipo. Todos estos concursosfueron premiados con puntos paralos equipos participantes ( los mismos equipos que se emplearon en clase), de forma que la afiliación se fomentó de forma lúdica, participativa y con puntuación. (4) Encuestas periódicas: Para reforzar el sentimiento de afiliación de 
los equipos, se fueron proponiendo encuestas en Facebook sobre el contenido tratado en clase: ( «vota ción para la coreografía más vistosa», «votación para elegir el estandarte de equipo mejor elaborado»). En los periodos de vigencia de las encuestas, los miembros de cada equipo iban votando por su coreografía o por su estandarte, involucrando a las familias y los estudiantes de los equipos, colaborando para conseguir puntos para susequipos.

3. Competición formal. A lo largo de todalatemporada, se celebraron cuatro momentos de competición: uno de habilidades gimnásticas individuales, otro sobre figuras de dúos, el tercero fue de figuras de tres componentes y la última competición fue el acto final de exhibición de la coreografía. Facebook fue utilizado para hacer de la competición un elemento educativo. Se desarrollaron las siguientes acciones: (a) Publicación de la preparación de la competición: A través de clips de video que mostraban los ensayos previos del alumnado para las competiciones. las practicas del alumnado previas a la competición proporcionar on un feedback sobre aspectos de la ejecución de las tareas y la posibilidad de mejorar a los estudiantes (Koekoek et al., 2018). (b) Publicación de los criterios, reglamentos de las competiciones: Anticipando el momento de cada competición se publicaron en el «muro» de Facebook los criterios que se aplicarían el día de las pruebas, compartiendo los objetivos de la actividad con el alumnado, (Zabala $\&$ Arnau 2007). (c) Publicación delasactividadescompetitivas, Lacompetición que se celebraba en el gimnasio fue grabada y publicada en Facebook. J unto a las habilidades motrices, se publicó, además la actividad del alumnado, que en las competiciones fueron desempeñando diferentes roles, como fotógrafos y jueces. Además de utilizar Facebook para acreditar el proceso educativo en el aula de EF y las competiciones del alumnado, se empleó para implementar la competición desde los hogares a través de actividades en las que los miembros de la familia podían contribuir siendo protagonistas de la participación, realizando actividad física desde Facebook y publicándola en la red social y obteniendo puntos por ello. Los mismos ejercicios propuestos en clase, u otros publica dos ad hoc para Facebook, fueron las «tareas» que real izaron y publicaron las familias.

4. Registro sistemático. En el MED, los resulta dos de las tareas propuestas se registran y se hacen públicos, no solo para determinar los niveles que los estudiantes han de alcanzar, sino también para informarles de su marcha a lo largo de toda la temporada proporcionando unaevaluación continuay el progreso de apren- dizaje hacia los objetivos deseados (Siedentop et al., 2019). La adaptación de la publicación de los resultados a Facebook se llevó a cabo a través de las siguientes acciones: (a) Publicación de los resultados obtenidos. Todos los resultados de las actividades de clase fueron tenidos en cuenta a través de una clasificación en la que se contemplaban diferentes aspectos, desde la corrección de la ejecución de las técnicas motrices, hasta el cumplimiento de las tareas de organización (manejo del material a lo largo de la sesión), higiene (equipación y ropa de cambio); es decir, las diferentes tareas asignadas a los estudiantes a través de los roles que debían ir cumpliendo a lo largo de la actividad. Esta clasificación tuvo una importancia creciente a lo largo del tiempo y tuvo incidencia en facetas como el sentido de pertenencia al equipo y la responsabilidad compartida para conseguir objetivos comunes; el hecho de conseguir puntos por la correcta realización detareas fue un incentivo motivador para el alumnado. De esta forma se promovió, con el uso de Facebook la posibilidad de diferentes maneras de participación, contribuyendo a la mejora de la motivación para continuar en la actividad de forma proactiva (Hastie, Ward, \& Brock, 2017). Además, los recursos que ofrece Facebook fueron aprovechados paradinamizar la actividad, haciendo que se pudieran conseguir puntos de diferentes formas, convirtiendo la actividad en una actividad social, más allá del componente competitivo. Para ello se llevaron a cabo estas iniciativas: (a) Encuestas puntuables: Durante el tiempo de desarrollo de la actividad se fueron lanzando encuestas mediante las cual es se podían conseguir puntos. Las encuestas se referían a algunos aspectos de las actividades de clase, por ejemplo, se trataba de votar por la coreografía más coordinada, 0 por el mejor vestuario, o por el mejor trofeo autoconstruido por el alumnado. (b) Tareas puntuablescon plazo: Se propusieron tareas específicas para que fueran desarrolladas desde Facebook. El enunciado de estas ta reas fue publicado en Facebook y los participantes las tuvieron que ejecutar para conseguir la puntuación. Algunos ejemplosfueron: hacerse unafoto con los dos pies en el aire y disfrazado el fin de semana de carnaval, realizar trofeos reciclados antes de un plazo de varios días, hacer actividades físicas similares a las desarrolla das en las sesiones del centro educativo. (c) Tareas de logro puntuables: Facebook permitió utilizar la puntuación - y la competición- como un acicate para la participa ción; se propusieron tareas con plazos más dilatados y puntuaciones «extra»; una de estas tareas fue la que retaba a conseguir que las familias de los equipos se inscribieran en Facebook, por ejemplo. (d) Puntos cooperativos: 
Este recurso permitió dar un sentido colectivo a la puntuación a lo largo de la actividad. Un ejemplo de puntuación cooperativa es publicar en Facebook una ima gen - habitualmente relacionada con el contenido de clase- y mandar imprimirla, dando un val or a cada ima gen presentada en clase por los hijos/ hijas; de esta forma, se fomenta la cooperación entre los estudiantes, dando la opción de poder presentar fotocopias de la imagen publicada. La puntuación obtenida de esta ma nera es parte del proceso educativo. La pauta para utilizar la puntuación en Facebook fue la de no excluir, proponiendo puntuaciones por tareas asequibles donde cada participante pudiera conseguir el objetivo de puntuar.

5. Ambiente festivo. Esta es una característica primaria del MED por dos razones: primera, porque el deporte es divertido en el mundo real y segunda, porque hacer divertida la enseñanza hace que la experienciaseamássignificativa paralosparticipantes. (Siedentop et al., 2019). Todo el proceso de publicar una tarea en Facebook, incluyendo ensayos, búsqueda deinformación, grabación y publicación del video por parte de losmiembros del grupo fomentó un ambiente lúdico y consiguió crear una atmósfera festiva entre los participantes. Este ambiente festivo se extendió a todos los miembros del grupo y no solo a los componentes de cada equipo; el hecho de publicar y compartir las publicaciones fomentó una autentica comunidad de aprendizaje (Wenger \& Trayner-Wenger, 2015) en torno a la tarea concreta y más allá en torno a toda la actividad extendida en el tiempo (Calderón et al., 2016). Los recursos que ofrece Facebook se utilizaron para promover el ambiente festivo que requiere el MED. Las herramientas que proporcionan el «botón like», los comentarios y los mensajes fueron determinantes a la hora de generar un ambiente de festividad en torno a la actividad, ya que los participantes daban importancia al número de likes, emoticonos o comentarios recibidos en sus publicaciones. La aportación con publicaciones de lo que se hacía en clase de EF, 0 de lo que se hacía desde casa participando a través de Facebook, generó un ambiente en el que cada participante podía contribuir y se dio un sentido social y festivo que difícilmente se hubiera conseguido sin el uso de Facebook (Foster, Linehan, Kirman, Lawson, \& James, 2010). A la hora de usar Facebook se consideraron varias pautas para lograr un ambiente festivo a lo largo de la actividad, que son las siguientes: (a) Actividades y competiciones de carácter social: se propusieron tareas para su real ización y publicación en Facebook, en las que siempre fue necesaria la cooperación de varias personas para poder realizarla, tareas en las que se pu- dieran conseguir puntos si las realizaban personas diferentes cada vez, tareas en las que era necesario desa rrollar un proceso para conseguir el objetivo (por ejemplo: hacerse una foto en determinado lugar de la vía pública relacionado con la actividad física y publicarlo posteriormente). (b) Posibilidad departicipar frecuentemente: Tanto de forma activa, a través de contenido propio elaborado por los participantes, como de interacción a tra vés de comentarios, likes, etc. fomentando la participa ción social (Alhenshiri et al., 2015). La decisión de dejar el canal abierto a la participación de participantes para que pudieran interactuar entre sí y con relación a la actividad de referencia, haciendo comentarios 0 a tra vés de likes en sus publicaciones incentivó la participa ción en el grupo de Facebook a lo largo del tiempo de duración de la actividad y fomentó un ambiente distendido. (c) Posibilidad de participar significativamente: lo cual quiere decir que cada interacción o publicación fue tenida en cuenta y que se tradujo en puntos para los equipos. Para ello se tuvieron en cuenta dichas interacciones a través de concursos que premiaban a los equipos que recibían más likes en determinadas acciones como por ejemplo el que se estableció para votar al equipo favorito de cada semana. Participar y conseguir puntos estimuló la participación en el grupo de Facebook, favoreció el seguimiento de la actividad a través de la red social y la hizo más dinámica. (d) Diversidad de tareas: a fin de fomentar al máximo la participación, se propusieron tareas diversas para su publicación en Facebook, tanto motrices como no motrices, de diferente natura leza y teniendo en cuenta el criterio de participación universal y respetando el nivel de exposición de la propia imagen en fotos 0 videos. La participación se extendía a la interacción a través de comentarios o likes en las publicaciones; todo este desarrollo permitió que na die se sintiera excluido de la actividad generada a través de Facebook.

6. Evento final. El momento másfestivo de todala actividad desarrollada con el MED es el evento final, donde «lo importante es que todos los estudiantes participen» (Siedentop et al., 2019). El uso de Facebook permitió ir preparando el evento final desde el inicio de la temporada, fijando la fecha del último acto con anticipación, ir dando información gráfica y visual de lo que se va a realizar en la celebración final y hacer coparticipes del proceso educativo a los estudiantes (Calderón et al., 2016). A la hora de replicar el evento final en Facebook se articularon las siguientes acciones: (a) Tareas específicas para el acto final: Se fomentó el compromiso con la actividad, a través de tareas específicas 
para el acto final como la construcción de trofeos con material reciclado (Méndez-Giménez, 2014) cuya ela boración y resultados pueden ser publicados en el muro de Facebook correspondiente (Bernabe-M artín, 2017). (b) Publicación de recursos el aborados para el acto final: Además, otros materiales, como diplomas, entradas o invitaciones se compartieron a través de Facebook. (c) Fomento de los roles activos para el desarrollo del acto final: La organización y celebración del evento final fue parte de la festividad que rodeó a toda la actividad; Facebook contribuyó a que los estudiantes participarán activamente en la organización del evento final, retrasmitiendo los actos (competiciones y últimos ensayos), haciendo entrevistas que fueron grabadas y colgadas en la red social (Bernabe-Martín, 2018b), a este fin se asignaron roles para el acto, como «corresponsales» y «reportero/ a grafico/ a». (d) Retransmisión de los actos finales: Las pruebas o competiciones y la entrega de premios fueron publicadas en el muro de Facebook y compartidas para la comunidad educativa; haciendo que el proceso de aprendizaje fuera más importante para el alumnado (Koekoek \& Van Hilvoorde, 2018).

\section{Decisiones de acción para la próxima pues- ta en práctica}

Las consideraciones para el uso de Facebook como recurso educativo complementario son las siguientes: (1) Deben ofrecerse entornos seguros para los participantes, en los que puedan desenvolverse con confianza conociendo la finalidad de la actividad y en cuanto al tratamiento de sus datos; por eso la configuración de la privacidad debe ser adecuada. (2) Hay que aprovechar la red para mostrar todo el proceso educativo, la metodología y las relaciones interpersonales del alumnado, para ello no hay que hacer de Facebook un escaparate de demostraciones, sino también los ensayos y el contexto de actividad. (3) Emplear las herramientas y el lenguaje propios de las redes sociales: videos cortos, significativos, pocapalabray títulos cortos (Cleary, 2017). (4) Facebook como recurso complementario facilitará que la metodología de la actividad se haga más participativa, yaquese podrácontribuir desdeFacebook. (Fernandez-Rio \& Bernabe-Martín, 2019).

\section{Conclusiones}

Como se ha mostrado, Facebook puede ser emplea do como un potente recurso complementario a las clases presenciales de EF. Puede ayudar a divulgar la plani- ficación de la temporada, proporcionar información sobre aspectos organizativos de la actividad, sobre aspectos metodológicos, sobre los recursos que se emplea rán, dando una visión de conjunto de toda la actividad. Facebook puede ayudar al docente, ya que puede proporcionar información más allá de clase, pudiendo emplear las clases presenciales en hacer más tarea motriz y dejando las explicaciones publicadas en la red social (André, 2018). Respecto al alumnado, Facebook puede ser un motivador de la actitud hacia la actividad. La afiliación al equipo puede reforzarse a través de las propuestas que se desarrollen para ser realizadas y publicadas en Facebook, y la puntuación puede ser un elemento motivador dentro de un ambiente donde se haga posible la participación de todos los miembros del grupo de la actividad.

\section{Referencias}

Akbari, E., Pilot,A., \& Robert-Jan Simons, P. (2015).Autonomy, competence, and relatedness in foreign language learning through Facebook. Computersin Human Behavior, 48, 126-134. https./ / doi.org/ 10.1016/ j.chb.2015.01.036

Alhenshiri,A., Badesh, H., \&Stephanidis, C. (2015). TheU seof Facebook asanAssistingTool inTeaching Computer Science Courses In H c I International 2015 - Poster' Extended Abstracts Pt li. https: / doi.org/ 10.1007/ 978-3-319-21383-5_37

André, M. (2018). Using social media in the Sport Education model. InA. Casey,V.A. Goodyear and K. M. Armour (eds.) Digital Technology in Physical Education (pp. 122-140). Routledge.

Balogh, Z., Munk, M., \&Turcani, M. (2011).Analysisof Student' Behaviour in theWeb-based Distance Learning Environment. Proceedingsof the2nd International Conferenceon Circuits, Systems, Communications\& Computers.

Bandura, A. (1997). Seff-efficacy:The exercise of control. NewYork: W.H. Freeman.

Bernabe-Martín, J. (2017). World ChampionshipsinA crosport «ovellanos2016.»

Bernabe-Martín,J. (2018a). Facebook and sporteducation: using theSocial network to promoteafamily Network of participative learning. In J. Fernandez-Rio, A. M endez-Gimenez and R. Sanchez (eds.) XI congreso internacional deactividadesfísicascooperativas (pp. 59-70).Avilés, Universidad de0 viedo.

Bernabe-Martín, J. (2018b). World Championships in Athetics «ovellanos2018». An experienceof Facebook integration in Bilingual Physical Education.

Bezemer, J., \& Kress, G. (2017).Young people, facebook, and pedagogy: Recognizing contemporary forms of multimodal text making In M. Knotopodis, C. Varvantakis and C. Wulf (eds.) GlobalYouth in Digital Trajectories (pp. 22-43). London: 
Routledge.

Calderón,A., López-Chicheri, I., Fernández-Río,J., \& Sinelnikov, O.A. (2016).Antonio: «i really want them to beengaged and learn»:Theuse of social mediainhigher eduction. InA.C asey, V. A. Goodyear and K. M. Armour (eds.) Digital Technologies and Learning in Physical Education: Pedagogical (pp. 86-103). London: Routledge

Casey, A., Goodyear, V. A., \& Armour, K. M. (2016). Digital technologiesand learning in physical education: Pedagogical cases InA. Casey, V.A. Goodyear and K. M. Armour (eds.) DigitalTechnologiesand Learning in Physical Education:Pedagogical Cases (pp 247-257). London. Routledge.

Cecchini,J.A., González, C., Méndez-Giménez,A., \& FernándezRío,J. (2011).Achievement goals, social goals, and motivational regulationsin physical education settings. Psicothema, 23(1), 51-57.

Cleary, . (2017). FacebookAnalytics:The0 nly GuideYou'll Ever Need. Retrieved June 5, 2019, from Razorsocial website: https: / / www. razor social.com/ facebook-analyticsreferenceguide/

Dzvapatsva, G. P., Mitrovic, Z., \& Dietrich,A. D. (2014). Useof social mediaplatformsfor improvingacademic performance atFurther Education andTraining colleges South African Journal of Information M anagement, 16(1), 1-7.

English, R., \&Duncan-Howell, J. (2008). Facebook goesto college: using social networkingtoolsto supportstudentsundertaking teaching practicum. Journal of O nlineLearning andTeaching,4(4), 596-601.

Erjavec, K. (2013). A prendizaje informal através de Facebook entre alumnos eslovenos. Comunicar, 21(41), 1-9. https./ / doi.org/ 10.3916/ C41-2013-11

Facebook, I. (n.d.). Legal terms.

Fernandez-Rio, J. , \& Bernabe-M artín, J. (2019). Facebook and sport education: mirroring the model at home to promote parental involvement. Sport, Education and Society,24(8), 814827. https:/ / doi.org/ 10.1080/ 13573322.2018.1470971

Fewkes, A. M., \& M cCabe, M. (2012). Facebook: LearningTool or Distraction? Journal of Digital Learning inTeacher Education, 28(3), 92-98. https:// doi.org/ 10.1080/ 21532974.2012.10784686

Foster, D., Linehan, C., Kirman, B., Lawson, S., \&James, G. (2010). M otivating physical activity at work: using persuasivesocial media for compeitivestep counting. Proceedings of the 14th International AcademicM indTrek Conference: EnvisioningFutureM edia Environments

Guijarro, E., Rocamora, I., Evangelio, C., \& GonzálezVíllora, S. (2020). El modelo de Educación Deportivaen España: una revisión sistemática. Retos, 38, 886-894. https:/ / doi.org/ 10.47197/ retos. v38i38. 77249

Gutierrez, D., Segovia,Yessica, Gárcia, L.M, Fernández, G. (2019). Integración del aprendizajeservicio en el modelo deeduca ción deportivacomo facilitador delatransiciónalaeducación secundaria. Publicaciones. Facultad de Educación y Humanidades Del CampusdeM eilla, 49(4), 87-108.

Hardman, K. (2008). Physical Education in Schools. A Global Perspective. Kinesiology, 40 (1), 5-28. https:/ / doi.org/ » «

Hastie, P.A., de0 jeda, D. M. \& \& Calderón,A. (2011).A review of research on sport education: 2004 to the present. Physical Education and Sport Pedagogy, 16(2), 103-132. https:/ / doi.org/ 10.1080/ 17408989.2010 .535202

Hastie, P.A., Ward, J. K., \& Brock, S. J. (2017). Effect of graded competition on student opportunities for participation and success rates during a season of Sport Education. Physical Education and Sport Pedagogy,22(3), 316-327. https:/ / doi.org/ 10.1080/17408989.2016.1203888

Junco, R. (2013). Comparing actual and self-reported measures of Facebook use. Computers in Human Behavior, 29(3), 626631. https:/ / doi.org/ 10.1016/ j.chb.2012.11.007

Junco, R. \& \& Cotten, S. R. (2012). No A 4 U:The relationship between multitasking and academic performance. Computers and Education, 59 (2), 505-514. https:/ / doi.org/ 10.1016/ j.compedu. 2011.12.023

Kent, M. (2016). Adding to the mix: Students use of Facebook groupsand blackboard discussionforumsin higher education. KnowledgeM anagement and ELearning, 8(3), 444-463.

Koekoek, J., van der Mars, H., van der Kamp,J.,Wainga,W., \& van Hilvoorde, I. (2018). Aligning Digital video technology. The Journal of Physical Education Recreation D ance, 89(1), 12-22.

Koekoek, J., \&Van Hilvoorde, I. (2018). Digital technology in physical education: Global perspectives. Routledge.

Kontopodis, M.,Varvantakis, C., \&Wulf, C. (2017). Global youth in digital trajectories. London. Routledge. https:/ / doi.org/ 10.4324/9781315303239

Kretschmann, R. (2017). Employingtablettechnologyfor video feedback in physical education swimming class. Journal of ELearning and Knowledge Society, 13(2), 103-115. https:/ / doi.org/ 10.20368/ 1971-8829/ 1322

Luguetti, C., Goodyear,V.A., \&André, M. H. (2019).'Thatislikea 24 hours-day tournament!' : using social mediato further an authentic sport experience within sport education. Sport, Education and Sociedy,24(1), 78-91. https:/ / doi.org/ 10.1080/ 13573322.2017.1292235

Madge, C., M eek, J.,Wellens, J., \& Hooley, T. (2009). Facebook, socia integration and informal learningat university: «ttismore for socialisingand talkingto friendsabout workthanfor actually doing work. » Learning, M edia andTechnology, 34 (2), 141-155. https: / doi.org/ 10.1080/ 17439880902923606

Maher, C. A., Lewis, L. K., Ferrar, K., Marshall, S., De Bourdeaudhuij, I., \& Vandelanotte, C. (2014). Are health behavior changeinterventionsthat useonlinesocia networks effective?A systematicreview. Journal of Medical Internet Research, 16(2), e40. https:/ / doi.org/ 10.2196/ jmir.2952

Margarit, E.-M.,Tonita, F., \&Angelescu, N. (2018). Employing Social MediainWorking withAthletes.A CaseStudy Discussing 
Ethica Implicationsfor Applied SportPsychology Consultants ELearning \& Softwarefor Education, 3, 308-313.

Meishar-Ta, H., Kurt, G. \& \& Pieterse, E. (2012). Facebookgroups asLM S:A casestudy. International Review of Rescarch in 0 pen and Distancelearning, 13(4), 33-48. https:/ / doi.org/ 10.19173/ irrodl. v13i4. 1294

Mena, G., Llupià,A., GarcíaBasteiro,A. L.,Aldea, M., Sequera,V.G., \&Trilla, A. (2012). TheW illingnessof Medical Studentsto U seFacebook asaTraining Channel for Professional Habits: TheCase of InfluenzaVaccination. Cyberpsychology, Behavior, and Social N etworking, 15(6), 328-331. https./ / doi.org/ 10.1089/ cyber.2011.0457

Méndez-Giménez,A. (2014). M odelosinnovadoresdeenseñanza de portiva en un contexto escolar. Propuesta dehibridación con el enfoque basado en la autoconstrucción demateriales.

Menendez-Santurio, J. I., \& Fernández-Río, J. (2016). Hibridación de los modelos deEducación Deportivay Responsabilidad Personal y Social: Unaexperienciaatravésdeun programade kickboxingeducativo. Retos 30, 113-121.

Menendez, J. I., \& Fernandez-Rio, J. (2017). Hybridising Sport EducationandTeachingfor Personal and Social Responsibility to includestudentswith disabilities. European Journal of Special N eedsEducation, 32 (4), 508-524. https./ / doi. org/ 10.1080/ 08856257.2016 .1267943

Mohammed, Z. (2016). The Impact of the Lack of Physical Education and SportsLessonon FitnessH eath-C aseFemale Students. European Journal of Physical Education and Sport Science, 1(2), 6-19.

Napolitano, M.A., Hayes, S., Bennett, G. G., Ives, A. K., \& Foster, G. D. (2013). U sing facebook and text messaging to deliver a weightlossprogram to collegestudents. Obesity,21(1), 25-31. https:/ / doi.org/ 10.1038/ oby.2012.107

Niu, L. (2017). UsingFacebook for A cademic Purposes: Current Literature and Directions for Future Research. Journal of Educational Computing Research 56(8), 1384-1406.

O mar, H., Embi, M.A. \&\& M dYunus, M. (2012). ESL learners' interaction in an online discussion via Facebook. Asian Social Science, 8(11), 67-74. https:// doi.org/ 10.5539/ ass. v8n11p67

Palade, T., Gheorghe, G., Ciolca, S.-M., \& Badea, D. (2018). Contribution of Social Networks to the Development of FootballTechnique. ELearning \& Softwarefor Education, 3, 314321.

Puente-M axera, F., Méndez-Giménez,A., \& M artínezde0 jeda, D. (2020). Games from around the world: Promoting intercultura competencethrough sporteducation in secondary school students. International Journal of Intercultural Redations, 75, 23-33. https:/ / doi.org/ 10.1016/ j.jintrel.2020.01.001

Queensand, E. D. (2018). Parentand Communityengagmentframework. Retrieved from http:/ / education.qld. gov.au/ schools/ parent-community-engagement-framework/ communication/
Romar,J. E., Sarén,J., \& Hastie, P. (2016).Athlete-centred coaching using the sport education model in youth soccer. Journal of Physical Education and Sport, 16(2), 380-391. https/ / doi.org/ 10.7752/ jpes.2016.02060

Sánchez-Bañuelos, F. (1997). La actividad físca orientada hacia la salud. Madrid: BibliotecaNueva.

Selwyn, N., \& Stirling, E. (2016). Social mediaand education... . now the dust hassettled. Learning,M edia andTechnology,41(1), 1-5. https. / doi.org/ 10.1080/ 17439884.2015.1115769

Servicio deInspecciónEducativadelaConsejeríadeEducacióny Cultura Informesobreutilización por partedeprofesorado yalumnado deaplicacionesquealmacenan datosen nubecon sistemasajenosa la plataforma educativa institucional del Gobierno del Principado de Asturias. (2018).

Siedentop, D. (1994). Sport education:Q uality PE through positivesport experiences. Champaign, IL: HumanK Kinetics.

Siedentop, D. L., Hastie, P.A., \&Van Der Mars, H. (2019). CompleteGuideto SportEducation. Champaign, IL: Human Kintics

Statista (2018). N umber of social mediausersworldwidefrom 2010 to 2021.

Stutzman, F. (2006). Anevaluation of identity-sharing behavior in social network communities. International Digital and Media Artss ournal. https:/ / doi.org/ 10.1.1.91.617

Wallhead, T., \& 0 'sullivan, M . (2005). Sport Education: physical education for thenew millennium?Physical Education \& Sport Pedagogy, 10(2), 181-210. https:/ / doi.org/ 10.1080/ 17408980500105098

Wang,Y. (2018).TheC ontext of CollegeStudents' FacebookU se andAcademic Performancel :AnEmpirical Study. Proceedings of the2018 CHI Conferenceon Human Factorsin Computing Systems. https:/ / doi.org/ 10.1145/ 3173574.3173992

Wenger, E. (2011). Communitiesof practice:A brief introduction. STEP LeadershipWorkshop, 1-7. https:/ / doi.org/ 10.1017/ CB0 9780511803932

Wenger, E., \&Trayner-Wenger, B. (2015). Communities of pratice: abriefintroduction. https:/ doi.org/ 10.2277/ 0521663636

Wood, E., Zivcakova, L., Gentile, P.,Archer, K., DePasquale, D., \& Nosko, A. (2012). Examining the impact of off-task multitasking with technology on real-time classroom learning Computersand Education, 58(1), 365-374. https: / / doi.org/ 10.1016/ j.compedu.2011.08.029

Wu, C.-H., \&Chen, S.-C. (2015). Understandingtherelationships of critical factors to Facebook educational usage intention. InternetResearch, 25(2), 262-278. https:/ / doi.org/ 10.1108/ IntR-11-2013-0232

Zabala,A., \&Arnau, L. (2007). Laenseñanzadelascompetencias Aula delnnovación Educativa, 161, 40-46.

Zhang,T., \& Li, H. (2018). Digita video and self-modelingin the PE classroom. InInA.Casey,V.A. Goodyear and K. M.Armour (eds.) Digital Technology in Physical Education (pp. 35-47). London: Routledge. 